\title{
Auction-Based Resource Allocation for Cooperative Communications
}

\author{
Jianwei Huang, Zhu Han, Mung Chiang, and H. Vincent Poor
}

\begin{abstract}
Distributed and efficient resource allocation is critical for fully realizing the benefits of cooperative communications in large scale communication networks. This paper proposes two auction mechanisms, the SNR auction and the power auction, that determine relay selection and relay power allocation in a distributed fashion. A single-relay network is considered first, and the existence and uniqueness of the Nash Equilibrium (i.e., the auction's outcome) are proved. It is shown that the power auction achieves the efficient allocation by maximizing the total rate increase, and the SNR auction is flexible in trading off fairness and efficiency. For both auctions, the distributed best response bid updates globally converge to the unique Nash Equilibrium in a completely asynchronous manner. The analysis is then generalized to networks with multiple relays, and the existence of the Nash Equilibrium is shown under appropriate conditions. Simulation results verify the effectiveness and robustness of the proposed algorithms.
\end{abstract}

Index Terms-Cooperative communications, game theory, auction theory, resource allocation, wireless networks

\section{INTRODUCTION}

C OOPERATIVE communications take advantage of the broadcast nature of wireless channels, uses relay nodes as virtual antennas, and thus realizes the benefits of multipleinput-multiple-output communications in situations where physical multiple antennas are difficult to deploy (e.g., small sensor nodes). Various cooperative protocols have been proposed (e.g., [1]-[5]), such as amplify-and-forward, decodeand-forward, and estimate-and-forward. Although the physical layer performance of cooperative communications has been extensively studied in the context of small networks from an information theoretic point of view, there are still many open problems in terms of how to realize its full benefits in large-scale networks through efficient resource allocation. In particular, such performance optimization requires the knowledge of global channel information (including that for sourcedestination, source-relay, and relay-destination channels) and heterogeneous resource constraints among users. Centralized

Manuscript received August 15, 2007; revised March 10, 2008. This work was supported by CUHK RGC Direct Grant C001-2050398, the NSF Grants ANI-03-38807, CCR-02-05214, CNS-06-25637, CCF-0448012, CCF0635034, and ONR YIP grants. Some preliminary results have been presented at the 2007 IEEE Global Communication Conference, Washington DC, November 2007, and at the 2008 IEEE International Conference on Acoustics, Speech, and Signal Processing, Las Vegas, Navada, April 2008.

J. Huang is with the Department of Information Engineering, The Chinese University of Hong Kong, Shatin, NT, HKSAR, China (e-mail: jwhuang@ie.cuhk.edu.hk).

Z. Han is with the University of Houston, Houston, Texas (e-mail: hanzhu22@gmail.com).

M. Chiang and H. V. Poor are with the Department of Electrical Engineering, Princeton University, Princeton, NJ, USA (e-mails: \{chiangm,poor\}@ princeton.edu).

Digital Object Identifier 10.1109/JSAC.2008.080919. information exchange and coordination, however, typically do not scale well with the network size This motivates our study of distributed resource allocation algorithms for cooperative communications.

Resource allocation in cooperative networks has attracted attention from the research community only recently. Related work in this area can be divided into two categories: centralized (e.g., [6], [7]) and distributed (e.g., [8]-[12]). For example, in [6], Nosratinia and Hunter proposed a centralized approach for grouping and partner selection to achieve maximal network-wide diversity. In [7], Ng and Yu designed centralized resource allocation algorithm for power control, bandwidth allocation, relay selection and relay strategy choice in an Orthogonal Frequency-Division Multiple Access (OFDMA) based relay network. Bletsas et al. in [8] proposed a distributed relay selection scheme, where one user chooses the "best" end-to-end path among many relays based on instantaneous channel measurements. In [9], Savazzi and Spagnolini considered distributed power control for a single user multiple hop transmission. Himsoon et al. in [10] investigated the relay selection and power management schemes for lifetime extension in wireless sensor networks. Annavajjala et al. in [11] studied the optimal power control problem for different cooperative protocols under high Signal to Interference-plusnoise Ratio (SINR) approximation for a single user and multiple relays. Wang et al. in [12] investigated resource allocation for cooperative transmission using Stackelberg games. However, the above work did not consider distributed resource allocation for multiple users (source-destination pairs) and multiple relays, where each user is allowed to use more than one than one relay and each relay can help more than one users.

In this paper, we focus on answering the following two questions: 1) "When to relay", i.e., when is it beneficial for a user to use the relay(s)? and 2) "How to relay", i.e., how should a user choose the relay(s) and how should each relay allocate its resource (transmission power) among multiple competing users? We address these two issues by designing an auction-based resource allocation framework. Auction theory [13] has recently been introduced to several areas of wireless communications (e.g., time slot allocation [14], power control [15], and cognitive radio networking [16]). To the best of our knowledge, this paper is the first work that applies auction theory in designing resource allocation schemes for cooperative communications.

We consider two network objectives: fairness and efficiency. Both are difficult to achieve even in a centralized fashion. This is because users' rate increases are non-smooth and 
non-concave in the relay's transmission power, and thus the performance optimization problem is non-convex. We propose two auction mechanism, the SNR auction and the power auction, which achieve the desired network objectives in a distributed fashion under certain technical conditions. In both auctions, each user decides "when to relay" based on a simple threshold policy that is locally computable. The question of "how to relay" is answered by a simple weighted proportional allocation among users who use the relay. The power auction achieves the efficiency allocation, and the SNR auction offers a flexible approach to achieve various tradeoffs between fairness and efficiency. Moreover, we show that the desirable outcomes of the auctions (i.e., Nash Equilibrium) can be achieved by users' greedy best response updates with local information in a completely asynchronous manner. The results are quite general and are applicable to wireless networks with multiple relays at different locations with different resource constraints. Simulation results demonstrate optimality, fairness, and convergence of the proposed auctions.

Next we present the system model and network objectives in Section II. In Section III, two auction mechanisms are proposed, their mathematical properties are analyzed, and the algorithms for achieving the Nash Equilibrium in a distributed fashion are shown. Extensions to the multiple relay case are discussed in Section IV. Simulation results are shown in Section V and we conclude in Section VI.

\section{System Model And Network Objectives}

\section{A. System Model}

We focus our discussions on the amplify-and-forward (AF) cooperation protocol [2] in this paper. Other cooperation protocols can be analyzed in a similar fashion. We begin by considering a simple system model as in Fig. 1, where there are one relay node $r$ and a set $\mathcal{I}=(1, \ldots, I)$ of sourcedestination pairs (i.e., users). Each user $i$ includes a source node $s_{i}$ and a destination node $d_{i}$. The case of multiple relays will be discussed in Section IV.

We assume that different users transmit their signals using non-overlapping frequency bands and thus they do not interfere with each other. For each user $i$, the cooperative transmission consists of two phases.

In Phase 1, source $s_{i}$ broadcasts its information so that it can be received by both destination $d_{i}$ and relay $r$, respectively, as

$$
Y_{s_{i}, d_{i}}=\sqrt{P_{s_{i}} G_{s_{i}, d_{i}}} X_{s_{i}}+n_{d_{i}},
$$

and

$$
Y_{s_{i}, r}=\sqrt{P_{s_{i}} G_{s_{i}, r}} X_{s_{i}}+n_{r}
$$

Here $P_{s_{i}}$ represents the fixed transmit power of source $s_{i}$, $X_{s_{i}}$ is the transmitted information symbol with unit energy at source $s_{i}, G_{s_{i}, d_{i}}$ and $G_{s_{i}, r}$ are the channel gains from $s_{i}$ to destination $d_{i}$ and relay $r$, respectively, and $n_{d_{i}}$ and $n_{r}$ are additive white Gaussian noises. Without loss of generality, we assume that the noise level is the same for all links and is denoted by $\sigma^{2}$. We also assume that the transmission frame length is small compared with the channel coherence time such that all channel gains are fixed during the time of interest. The
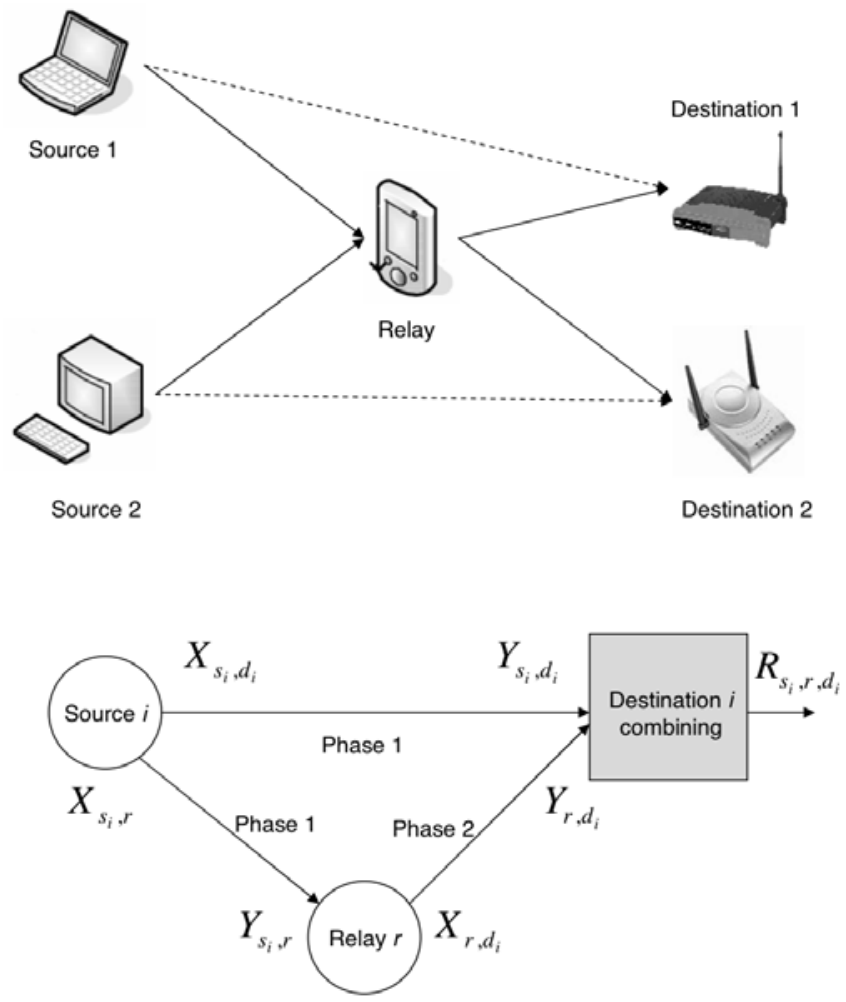

Fig. 1. System model for cooperative transmission (single relay case)

signal-to-noise ratio (SNR) obtained at destination $d_{i}$ in Phase 1 is

$$
\Gamma_{s_{i}, d_{i}}=\frac{P_{s_{i}} G_{s_{i}, d_{i}}}{\sigma^{2}} .
$$

In Phase 2, relay $r$ amplifies $Y_{s_{i}, r}$ with power $P_{r, d_{i}}$ and forwards it to destination $d_{i}$. The received signal at destination $d_{i}$ in Phase 2 is

$$
Y_{r, d_{i}}=\sqrt{P_{r, d_{i}} G_{r, d_{i}}} X_{r, d_{i}}+n_{d_{i}}^{\prime}
$$

where

$$
X_{r, d_{i}}=\frac{Y_{s_{i}, r}}{\left|Y_{s_{i}, r}\right|}
$$

is the unit-energy transmitted signal that relay $r$ receives from source $s_{i}$ in Phase $1, G_{r, d_{i}}$ is the channel gain from relay $r$ to destination $d_{i}$, and $n_{d_{i}}^{\prime}$ is the received noise at Phase 2. Substituting (2) into (5), we can rewrite the received signal (4) as

$$
Y_{r, d_{i}}=\frac{\sqrt{P_{r, d_{i}} G_{r, d_{i}}}\left(\sqrt{P_{s_{i}} G_{s_{i}, r}} X_{s_{i}, d_{i}}+n_{r}\right)}{\sqrt{P_{s_{i}} G_{s_{i}, r}+\sigma^{2}}}+n_{d_{i}}^{\prime} .
$$

Using (6), the relayed SNR at destination $d_{i}$ in Phase 2 is

$$
\Gamma_{s_{i}, r, d_{i}}=\frac{P_{r, d_{i}} P_{s_{i}} G_{r, d_{i}} G_{s_{i}, r}}{\sigma^{2}\left(P_{r, d_{i}} G_{r, d_{i}}+P_{s_{i}} G_{s_{i}, r}+\sigma^{2}\right)} .
$$

Now we are ready to calculate the achievable rate at destination $d_{i}$. At a given transmission time, user $i$ has two choices:

1) Use Phase 1 only and achieve rate (in $\mathrm{bps} / \mathrm{Hz}$ )

$$
R_{s_{i}, d_{i}}=W \log _{2}\left(1+\Gamma_{s_{i}, d_{i}}\right)
$$

where $W$ is the signal bandwidth. 


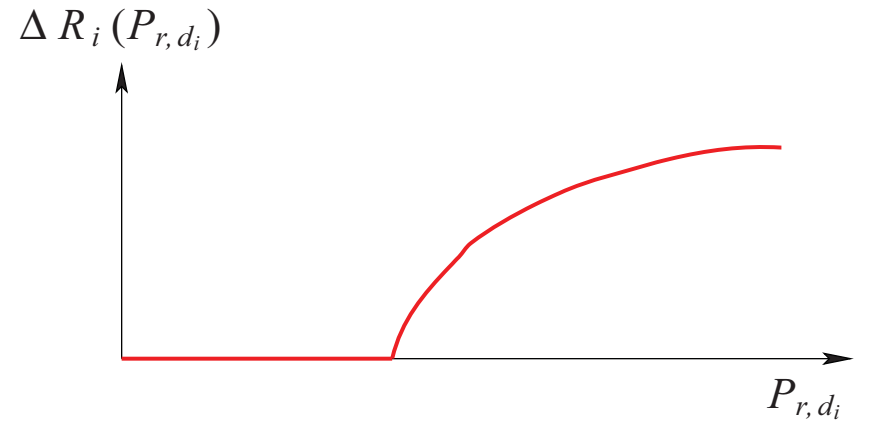

Fig. 2. Rate increase as a function of relay transmission power

2) Use both phases and achieve rate (at the output of maximal ratio combining)

$$
R_{s_{i}, r, d_{i}}=\frac{1}{2} W \log _{2}\left(1+\Gamma_{s_{i}, d_{i}}+\Gamma_{s_{i}, r, d_{i}}\right) .
$$

The coefficient $1 / 2$ is due to the fact that cooperative transmission uses half of the resources (e.g., time slots, frequency bands, orthogonal codes). Since $\Gamma_{s_{i}, r, d_{i}}$ is the extra SNR increase compared with the direct transmission, we also denote

$$
\triangle \mathrm{SNR}_{i} \triangleq \Gamma_{s_{i}, r, d_{i}}
$$

Comparing these two choices, the rate increase that user $i$ can obtain comparing with the case of no relay is

$$
\triangle R_{i}=\max \left\{R_{s_{i}, r, d_{i}}-R_{s_{i}, d_{i}}, 0\right\},
$$

which is nonnegative since the source can always choose not to use the relay and thereby obtain zero rate increase. $\triangle R_{i}$ is a function of the channel gains of the source-destination, sourcerelay and relay-destination links, as well as the transmission power of the source and the relay. In particular, $\triangle R_{i}$ is a non-decreasing, non-smooth, and non-concave function of the relay transmission power $P_{r, d_{i}}$, as illustrated in Fig. 2.

We assume that the source transmission power $P_{s_{i}}$ (for all users $i$ ) and the relay's total power $P$ are fixed. The relay determines the allocation of its transmission power among users, $\boldsymbol{P}_{r} \triangleq\left(P_{r, d_{i}}, \forall i \in \mathcal{I}\right)$, such that the total power constraint is not violated, i.e.,

$$
\boldsymbol{P}_{r} \in \mathcal{P}_{r} \triangleq\left\{\boldsymbol{P}_{r} \mid \sum_{i \in \mathcal{I}} P_{r, d_{i}} \leq P, P_{r, d_{i}} \geq 0, \forall i \in \mathcal{I}\right\} .
$$

\section{B. Network Objectives: Efficiency and Fairness}

We consider two different network objectives: efficiency and fairness. An efficient power allocation $\boldsymbol{P}_{r}^{\text {efficient }}$ maximizes the total rate increase of all users, i.e. solves the following problem,

$$
\max _{\boldsymbol{P}_{r} \in \mathcal{P}_{r}} \sum_{i \in \mathcal{I}} \triangle R_{i}\left(P_{r, d_{i}}\right) .
$$

In many cases, an efficient allocation discriminates against users who are far away from the relay. To avoid this, we also consider a fair power allocation $\boldsymbol{P}_{r}^{\text {fair }}$, which solves the following problem,

$$
\begin{array}{ll}
\min _{\boldsymbol{P}_{r} \in \mathcal{P}_{r}} & c \\
\text { subject to } & \frac{\triangle R_{i}\left(\triangle \mathrm{SNR}_{i}\right)}{\partial\left(\triangle \mathrm{SNR}_{i}\right)}=c q_{i} \cdot \mathbf{1}_{\left\{\triangle \mathrm{SNR}_{i}>0\right\}}, \forall i \in \mathcal{I} .
\end{array}
$$

Here $1_{\{\cdot\}}$ is the indicator function, and $q_{i}$ is a user dependent priority parameter. When $q_{i}=1$ for each $i$, all users who use the relay will have the same marginal utility, which leads to strict fairness among users. ${ }^{1}$ It is possible to assign different weights to different users to achieve different Qualities of Service. One such example is to let

$$
q_{i}=\frac{W}{2 \ln 2\left(1+\Gamma_{s_{i}, d_{i}}\right)}=\left.\frac{\partial \triangle R_{i}\left(\triangle \mathrm{SNR}_{i}\right)}{\partial\left(\triangle \mathrm{SNR}_{i}\right)}\right|_{\triangle \mathrm{SNR}_{i}=0},
$$

i.e., $q_{i}$ represents user $i$ 's eagerness to gain extra SNR increase by using the relay. The intuition behind Problem (14) is that for all users that choose to use the relay, the corresponding $\triangle$ SNR should be maximized subject to the "weighted marginal utility equalization" condition. This can be translated into the minimization of the common coefficient $c$ due to the concavity of $\triangle R_{i}$ in terms of $\triangle \mathrm{SNR}_{i}$ in the proper regime. Numerical examples for both equal weights and different weights are shown in Section V.

We notice that a fair or efficient allocation is Pareto optimal, i.e., no user's rate can be further increased without decreasing the rate of another user. However, an efficient or fair allocation need not fully utilize the resources at the relay, i.e., $\sum_{i \in \mathcal{I}} P_{r, d_{i}}$ can be less than $P$. This could happen, for example, when the relay is far away from all users so that allowing the relay to transmit half of the time will only decrease the total achievable rate. This is very different from most previous network resource allocation problems (e.g. [16]), in which the network performance is maximized only if the resource is fully utilized.

Since $\triangle R_{i}\left(P_{r, d_{i}}\right)$ is non-smooth and non-concave, both Problems (13) and (14) are difficult to solve even in a centralized fashion. In the rest of the paper, we will propose two auction mechanisms, and quantify the technical conditions under which the auction mechanisms solve the above problems in a distributed fashion.

\section{Auction Mechanisms}

An auction is a decentralized market mechanism for allocating resources. The essence of an auction is a game, where the players are the bidders, the strategies are the bids, and both allocations and payments are functions of the bids. One well known auction is the Vickrey-Clarke-Groves (VCG) auction [13], which requires gathering global information from the network and performing centralized computations. To overcome the limitation of the VCG auction, we propose

\footnotetext{
${ }^{1}$ Most work on cooperative networks is based on information theoretic analysis, with the focus on calculating the achievable capacity (i.e., users' rate increases). Although we follow the same tradition in this paper, the proposed algorithms can be used to achieve other network objectives, such as $\alpha$-fairness, that are more commonly used in the networking community.
} 
two simple auctions, the SNR auction and the power auction, both of which have simple rules as described next. ${ }^{2}$

\section{SNR Auction and Power Auction}

- Information: Besides the public and local information (i.e., $W, P, \sigma^{2}, P_{s_{i}}, G_{s_{i}, d_{i}}$ ), each user $i$ also knows the channel gains $G_{s_{i}, r}$ and $G_{r, d_{i}}$, through feedback from relay $r$. The relay announces a positive reserve bid $\beta>0$ and a price $\pi>0$ to all users before the auction starts.

- Bids: User $i$ submits a scalar $b_{i} \geq 0$ to the relay.

- Allocation: The relay allocates transmit power according to

$$
P_{r, d_{i}}=\frac{b_{i}}{\sum_{j \in \mathcal{I}} b_{j}+\beta} P \text {. }
$$

- Payments: In an SNR auction, source $i$ pays the relay $C_{i}=\pi q_{i} \triangle \mathrm{SNR}_{i}$, where $q_{i}$ is a user-specific parameter. In a power auction, source $i$ pays the relay $C_{i}=\pi P_{r, d_{i}}$.

A bidding profile is defined as the vector containing the users' bids, $\boldsymbol{b}=\left(b_{1}, \ldots, b_{I}\right)$. The bidding profile of user $i$ 's opponents is defined as $\boldsymbol{b}_{-i}=\left(b_{1}, \ldots, b_{i-1}, b_{i+1}, \ldots, b_{I}\right)$, so that $\boldsymbol{b}=\left(b_{i} ; \boldsymbol{b}_{-i}\right)$. User $i$ chooses $b_{i}$ to maximize its payoff

$$
U_{i}\left(b_{i} ; \boldsymbol{b}_{-i}, \pi\right)=\triangle R_{i}\left(P_{r, d_{i}}\left(b_{i} ; \boldsymbol{b}_{-i}\right)\right)-C_{i}\left(b_{i} ; \boldsymbol{b}_{-i}, \pi\right) .
$$

For notational simplicity, we omit the dependence on $\beta$ and other system parameters.

If the reserve bid $\beta=0$, then the resource allocation in (16) depends only on the ratio of the bids. A bidding profile $k \boldsymbol{b}$ (for any $k>0$ ) leads to the same resource allocation as $\boldsymbol{b}$, which is not desirable in practice. That is why we need a positive reserve bid. However, the value of $\beta$ is not important as long as it is positive. For example, if we increase $\beta$ to $k^{\prime} \beta$, then users can simply scale $\boldsymbol{b}$ to $k^{\prime} \boldsymbol{b}$ (for any $k^{\prime}>0$ ), which leads to the same resource allocation. For simplicity, we choose $\beta=1$ in all the simulations in Section $\mathrm{V}$.

The desirable outcome of an auction is called a Nash Equilibrium (NE), which is a bidding profile $\boldsymbol{b}^{*}$ such that no user wants to deviate unilaterally, i.e.,

$$
U_{i}\left(b_{i}^{*} ; \boldsymbol{b}_{-i}^{*}, \pi\right) \geq U_{i}\left(b_{i} ; \boldsymbol{b}_{-i}^{*}, \pi\right), \forall i \in \mathcal{I}, \forall b_{i} \geq 0 .
$$

Define user $i$ 's best response (for fixed $\boldsymbol{b}_{-i}$ and price $\pi$ ) as

$$
\mathcal{B}_{i}\left(\boldsymbol{b}_{-i}, \pi\right)=\left\{b_{i} \mid b_{i}=\arg \max _{\tilde{b}_{i} \geq 0} U_{i}\left(\tilde{b}_{i} ; \boldsymbol{b}_{-i}, \pi\right)\right\},
$$

which in general could be a set. An NE is also a fixed point solution of all users' best responses. We would like to answer the following four questions for both auctions: 1) When does an NE exist? 2) When is the NE unique? 3) What are the properties of the NE? 4) How can we reach the NE in a distributed fashion?

\footnotetext{
${ }^{2}$ Both auctions are similar to the ones proposed in [16]. However, here we consider the problem of relay resource allocation in a multiple-hop wireless network, which is different from the sing-hop wireless network considered in [16]. For example, due to the unique characteristics of the relay network, especially the non-smooth and non-concave nature of the rate increase function (e.g., Fig. 2), calculating the best response functions here demands a completely new method and leads to a very different threshold policy. We also consider the multiple relay case in Section IV, whereas [16] considers the problem of allocating resource only at a single measurement point.
}

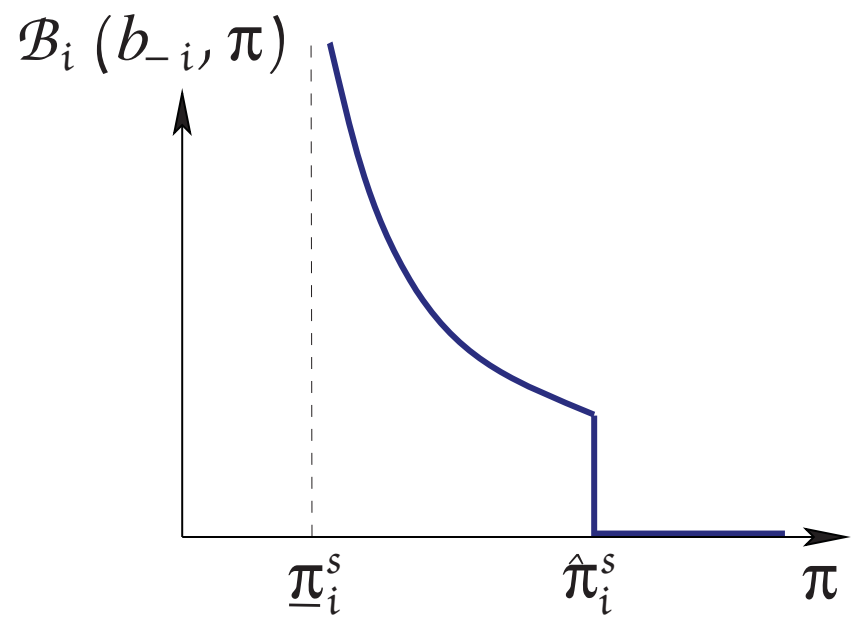

Fig. 3. User $i$ 's best response in an SNR auction if $\underline{\pi}_{i}^{s}<\hat{\pi}_{i}^{s}$.

\section{A. SNR Auction}

Let us first study the users' best responses (e.g., (19)) in the SNR auction. For each user $i$, there exist two critical price values, $\underline{\pi}_{i}^{s}$ and $\hat{\pi}_{i}^{s}$, where

$$
\underline{\pi}_{i}^{s} \triangleq \frac{W}{2 q_{i} \ln 2\left(1+\Gamma_{s_{i}, d_{i}}+\frac{P G_{r, d_{i}} P_{s_{i}} G_{s_{i}, r}}{\left(P_{s_{i}} G_{s_{i}, r}+P G_{r, d_{i}}+\sigma^{2}\right) \sigma^{2}}\right)},
$$

and $\hat{\pi}_{i}^{s}$ is the smallest positive root of

$$
\begin{aligned}
g_{i}^{s} & (\pi) \triangleq \pi q_{i}\left(1+\Gamma_{s_{i}, d_{i}}\right) \\
& -\frac{W}{2}\left(\log _{2}\left(\frac{2 \pi q_{i} \ln 2}{W}\left(1+\Gamma_{s_{i}, d_{i}}\right)^{2}\right)+\frac{1}{\ln 2}\right) .
\end{aligned}
$$

Function $g_{i}^{s}(\pi)$ represents the maximum payoff a user can achieve if it decides to use the relay. It is clear that when $g_{i}^{s}(\pi)<0$, the user is better off by not using the relay. Both $\underline{\pi}_{i}^{s}$ and $\hat{\pi}_{i}^{s}$ can be calculated based on local information (i.e., no information exchange is needed among different users), and one could be larger than the other depending on the specific system parameters.

Theorem 1: In an SNR auction, user $i$ 's unique best response function is

$$
\mathcal{B}_{i}\left(\boldsymbol{b}_{-i}, \pi\right)=f_{i}^{s}(\pi)\left(\sum_{j \neq i} b_{j}+\beta\right) .
$$

If $\hat{\pi}_{i}^{s}>\underline{\pi}_{i}^{s}$, then (23). If $\hat{\pi}_{i}^{s} \leq \underline{\pi}_{i}^{s}$, then $f_{i}^{s}(\pi)=\infty$ for $\pi<\hat{\pi}_{i}^{s}$ and $f_{i}^{s}(\pi)=0$ for $\pi \geq \hat{\pi}_{i}^{s}$.

Let us consider the intuition behind (22) and (23). First consider the case where $\hat{\pi}_{i}^{s}>\underline{\pi}_{i}^{s}$, in which case $\mathcal{B}_{i}\left(\boldsymbol{b}_{-i}, \pi\right)$ is illustrated in Fig. 3. The price $\hat{\pi}_{i}^{s}$ determines when it is beneficial for user $i$ to use the relay. With a price larger than $\hat{\pi}_{i}^{s}$, user $i$ cannot obtain a positive payoff from the auction no matter what bid it submits; thus it should simply use direct transmission and achieve a rate of $R_{s_{i}, d_{i}}$. As a result, $\mathcal{B}_{i}\left(\boldsymbol{b}_{-i}, \pi\right)$ is discontinuous at $\hat{\pi}_{i}^{s}$. When $\pi \in\left(\underline{\pi}_{i}^{s}, \hat{\pi}_{i}^{s}\right)$, user $i$ wants to participate in the auction, and its best response depends how much other users bid $\left(\boldsymbol{b}_{-i}\right)$. When the price is smaller than $\underline{\pi}_{i}^{s}$, user $i$ becomes so aggressive that it demands 


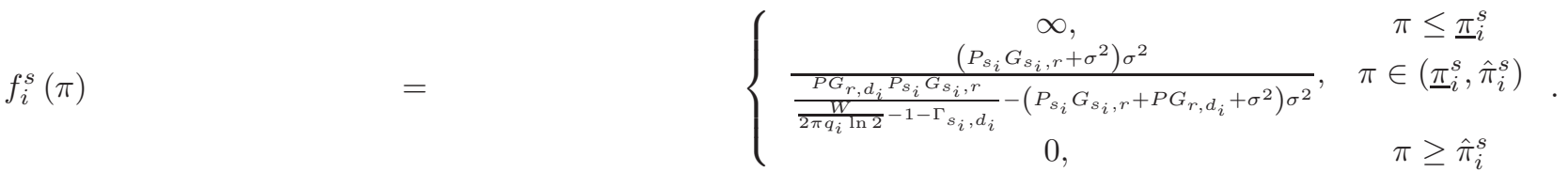

a large SNR increase that cannot be achieved even if all the resource is allocated to it. This is reflected by an infinite bid in (23). Next we consider the second case where $\hat{\pi}_{i}^{s} \leq \underline{\pi}_{i}^{s}$. In this case, user $i$ either cannot obtain a positive payoff or cannot achieve the desired SNR increase, and thus the best response is either 0 or $\infty$. A detailed proof of Theorem 1 can be found in Appendix A.

Combining (16) and (22), we know that if an NE exist, the relay power allocated for user $i$ is

$$
P_{r, d_{i}}(\pi)=\frac{f_{i}^{s}(\pi)}{f_{i}^{s}(\pi)+1} P,
$$

and $\sum_{i \in \mathcal{I}} \frac{f_{i}^{s}(\pi)}{f_{i}^{s}(\pi)+1}<1$. The strict inequality is due to the positive reserve bid $\beta$, and later we will show that the resulting resource waste can be made arbitrarily small under appropriate conditions.

Next, we need to find the fixed point of all users' best responses, i.e., the NE. A trivial case would be $\hat{\pi}_{i}^{s} \leq \underline{\pi}_{i}^{s}$ for all users $i$, in which case there exists a unique all-zero $\mathrm{NE}$ $\boldsymbol{b}^{*}=\mathbf{0}$ when $\pi \geq \max _{i} \hat{\pi}_{i}^{s}$. The more interesting case would be the following.

Definition 1: A network is SNR-regular if there exists at least one user $i$ such that $\hat{\pi}_{i}^{s}>\underline{\pi}_{i}^{s}$.

In an SNR-regular network, at least one user is interested in using the relay. A randomly generated network with many users and a relay will be SNR-regular with high probability, unless all users have good direct gains or the relay is far away from the users.

Theorem 2: Consider an SNR auction in an SNR-regular network. There exists a threshold price $\pi_{t h}^{s}$ such that a unique NE exists if $\pi>\pi_{t h}^{s}$; otherwise, no NE exists.

A proof of Theorem 2 is given in Appendix B. Unlike the results in [16], the unique $\mathrm{NE}$ in Theorem 2 might not be a continuous function of $\pi$, due to the discontinuity of the best response function as shown in Fig. 3. This has been observed in the simulation results described in Section V. In particular, the unique NE could be all zero for any price $\pi>\pi_{t h}^{s}$, even if the network is SNR-regular.

It can be seen that the "weighted marginal utility equalization" property of a fair allocation (i.e., the constraint in Problem (14)) is satisfied at the NE of the SNR auction. However, there is always some "resource waste" due to the positive reserve bid $\beta$ (i.e., some power will never be allocated to any user). Nevertheless, by choosing a price $\pi$ larger than but very close to $\pi_{t h}^{s}$, we could reduce the resource waste to a minimum and approximate the fair allocation. Formally, we define a reduced feasible set parameterized by $\delta$ as

$$
\mathcal{P}_{r}^{\delta} \triangleq\left\{\boldsymbol{P}_{r} \mid \sum_{i \in \mathcal{I}} P_{r, d_{i}} \leq P(1-\delta), P_{r, d_{i}} \geq 0, \forall i \in \mathcal{I}\right\} .
$$

Theorem 3: Consider an SNR auction in an SNR-regular network, where $f_{i}^{s}(\pi)$ is continuous at $\pi_{t h}^{s}$ for each user $i$, and positive and finite for at least one user. There exists a $\delta^{\prime}>0$ such that for any $0<\delta<\delta^{\prime}$, there exists a price $\pi^{s, \delta}$ under which the unique NE achieves the fair allocation $\boldsymbol{P}_{r}^{\text {fair }}$ with a reduced feasible set $\mathcal{P}_{r}^{\delta}$.

A proof is given in Appendix C. A sufficiently small $\delta$ makes sure that we deal with a regime in which $f_{i}^{s}(\pi)$ is continuous for all users. This is also desirable in practice since we want to minimize the amount of resources wasted.

Finally, we want to mention that the threshold price $\pi_{t h}^{s}$ is difficult to find analytically. This means that the price $\pi$ needs to be adjusted through a "trial-and-error" process. In particular, the price needs to be increased when users' bids are too large and decreased when the resource waste due to $\beta$ is too large. Since users' best responses in (23) are monotonically decreasing in price, a bi-section search of the price can lead to the desirable outcome.

\section{B. Power Auction}

For a power auction, we can also derive a closed-form solution for the best response functions, which are much more complicated compared with those for the SNR auction. To simplify the notations, we define $\tilde{P}_{s_{i}, r} \triangleq P_{s_{i}} G_{s_{i}, r}$. Furthermore, we define two functions $\left(z_{i}(\pi)\right.$ and $\left.g_{i}^{p}(\pi)\right)$ and two critical prices $\left(\underline{\pi}_{i}^{p}\right.$ and $\left.\bar{\pi}_{i}^{p}\right)$ in (26) to (29) (where (26) and (27) are located at the top of the following page):

$$
\bar{\pi}_{i}^{p} \triangleq \frac{W P_{s_{i}} G_{r, d_{i}} G_{s_{i}, r}}{2 \sigma^{2} \ln 2\left(\tilde{P}_{s_{i}, r}+\sigma^{2}\right)\left(1+\Gamma_{s_{i}, d_{i}}\right)},
$$

and

$$
\begin{array}{r}
g_{i}^{p}(\pi)=\frac{W}{2} \log _{2}\left(1+\Gamma_{s_{i}, d_{i}}+\frac{z_{i}(\pi) \tilde{P}_{s_{i}, r}}{\sigma^{2}\left(z_{i}(\pi)+\tilde{P}_{s_{i}, r}+\sigma^{2}\right)}\right) \\
-R_{s_{i}, d_{i}}-\pi \frac{z_{i}(\pi)}{G_{r, d_{i}}} .
\end{array}
$$

Proposition 1: $g_{i}^{p}(\pi)$ is strictly decreasing in $\pi \in\left[\underline{\pi}_{i}^{p}, \bar{\pi}_{i}^{p}\right]$.

A proof is given in Appendix D. It can also be shown that $g_{i}^{p}(0)=\frac{W}{2} \log _{2}\left(1+\Gamma_{s_{i}, d_{i}}+\frac{P_{s_{i}} G_{s_{i}, r}}{\sigma^{2}}\right)-R_{s_{i}, d_{i}}$ and $g_{i}^{p}\left(\bar{\pi}_{i}^{p}\right)<0$. If $g_{i}^{p}(0)>0$, then there exists a unique root for $g_{i}^{p}(\pi)$ within $\left(0, \bar{\pi}_{i}^{p}\right]$, and we denote it as $\hat{\pi}_{i}^{p}$. If $g_{i}^{p}(0) \leq 0$, we can simply let $\hat{\pi}_{i}^{p}=0$. Then we have the following result:

Theorem 4: In a power auction, user $i$ 's unique best response function is

$$
\mathcal{B}_{i}\left(\boldsymbol{b}_{-i}, \pi\right)=f_{i}^{p}(\pi)\left(\sum_{j \neq i} b_{j}+\beta\right) .
$$




$$
\begin{gathered}
z_{1, i}=\left(\tilde{P}_{s_{i}, r}+\sigma^{2}\right)\left(\tilde{P}_{s_{i}, r}+2 \sigma^{2}\left(1+\Gamma_{s_{i}, d_{i}}\right)\right) \\
z_{2, i}(\pi)=4\left(\tilde{P}_{s_{i}, r}+\sigma^{2}\left(1+\Gamma_{s_{i}, d_{i}}\right)\right)\left(\tilde{P}_{s_{i}, r}+\sigma^{2}\right)\left(\frac{W}{2 \pi \ln 2} \tilde{P}_{s_{i}, r} G_{r, d_{i}}-\sigma^{2}\left(\tilde{P}_{s_{i}, r}+\sigma^{2}\right)\left(1+\Gamma_{s_{i}, d_{i}}\right)\right), \\
z_{i}(\pi)=\frac{-z_{1, i}+\sqrt{z_{1, i}^{2}+z_{2, i}(\pi)}}{2\left(\tilde{P}_{s_{i}, r}+\sigma^{2}\left(1+\Gamma_{s_{i}, d_{i}}\right)\right)} .
\end{gathered}
$$

$$
\underline{\pi}_{i}^{p} \triangleq \frac{W \tilde{P}_{s_{i}, r} G_{r, d_{i}}\left(P_{s_{i}} G_{s_{i}, r}+\sigma^{2}\right)}{2 \ln 2\left[\sigma^{2}\left(1+\Gamma_{s_{i}, d_{i}}\right)\left(P G_{r, d_{i}}+\tilde{P}_{s_{i}, r}+\sigma^{2}\right)+\tilde{P}_{s_{i}, r} P G_{r, d_{i}}\right]\left(P G_{r, d_{i}}+\tilde{P}_{s_{i}, r}+\sigma^{2}\right)}
$$

If $\hat{\pi}_{i}^{p}>\underline{\pi}_{i}^{p}$, then

$$
f_{i}^{p}(\pi)=\left\{\begin{array}{cc}
\infty, & \pi<\underline{\pi}_{i}^{p} \\
\frac{z_{i}(\pi)}{P G_{r, d_{i}}-z_{i}(\pi)}, & \pi \in\left[\underline{\pi}_{i}^{p}, \hat{\pi}_{i}^{p}\right] . \\
0, & \pi>\hat{\pi}_{i}^{p}
\end{array} .\right.
$$

If $\hat{\pi}_{i}^{p} \leq \underline{\pi}_{i}^{p}$, then $f_{i}^{p}(\pi)=\infty$ for $\pi<\hat{\pi}_{i}^{p}$ and $f_{i}^{p}(\pi)=0$ for $\pi \geq \hat{\pi}_{i}^{p}$.

The proof of Theorem 4 is similar to that of Theorem 1 and thus is omitted due to space limitations. Theorem 4 is applicable for the general SNR regime. In the case of low SNR, i.e., when $\Gamma_{s_{i}, d_{i}}$ and $\triangle \operatorname{SNR}_{i}\left(b_{i}, \boldsymbol{b}_{-i}\right)$ are small for all $i$, we have

$$
\begin{aligned}
W \log _{2}\left(1+\Gamma_{s_{i}, d_{i}}\right. & \left.+\triangle \operatorname{SNR}_{i}\left(b_{i}, \boldsymbol{b}_{-i}\right)\right) \\
& \approx \frac{W}{\ln 2}\left(\Gamma_{s_{i}, d_{i}}+\triangle \operatorname{SNR}_{i}\left(b_{i}, \boldsymbol{b}_{-i}\right)\right) .
\end{aligned}
$$

In this case, we can show that $\hat{\pi}_{i}^{p}=0$ when $G_{s_{i}, d_{i}}>G_{s_{i}, r}$. In other words, when the direct channel gain between source and destination is larger than the channel gain between source and relay, a user will never use the relay in the low SNR regime.

In terms of the existence, uniqueness and properties of the $\mathrm{NE}$, we have the following results, which are similar in spirit to Theorems 2 and 3 for the SNR auction. The detailed proofs are omitted.

Definition 2: A network is power-regular if $\hat{\pi}_{i}^{p}>\underline{\pi}_{i}^{p}$ for at least one user $i$.

Theorem 5: Consider a power auction in a power-regular network. There exists a threshold price $\pi_{t h}^{p}>0$ such that a unique NE exists if $\pi>\pi_{t h}^{p}$; otherwise, no NE exists.

Theorem 6: Consider a power auction in a power-regular network, where $f_{i}^{p}(\pi)$ is continuous at $\pi_{t h}^{p}$ for each user $i$, and positive and finite for at least one user. There exists a $\delta^{\prime}>0$ such that for any $0<\delta<\delta^{\prime}$, there exists a price $\pi^{p, \delta}$ under which the unique $\mathrm{NE}$ achieves the efficient allocation $\boldsymbol{P}_{r}^{\text {efficient }}$ with a reduced feasible set $\mathcal{P}_{r}^{\delta}$.

We can see that for both auctions, the conditions for the existence, uniqueness, and effectiveness (fairness for SNR auction and efficiency for power auction) are quite similar. This is due to the linearity of the best response functions in both cases, which again is due to the weighted proportional allocation rule of the relay power in (16).

\section{Distributed Iterative Best Response Updates}

The last issue we want to address is how the NE can be reached in a distributed fashion. It is clear that the best response function in (23) can be calculated in a distributed fashion with limited information feedback from the relay. However, each user does not have enough information to calculate the best responses of other users, which prevents it from directly calculating the NE. Nevertheless, the NE can be achieved in a distributed fashion if we allow the users to iteratively update their bids based on best response functions in an asynchronous fashion.

Let us first consider the synchronous updates, where users update their bids $\boldsymbol{b}(t)$ at time $t$ according to the best response functions $b_{i}(t)=\mathcal{B}_{i}\left(\boldsymbol{b}_{-i}(t-1), \pi\right)$, based on other users' bids $\boldsymbol{b}_{-i}(t-1)$ at time $t-1$. Taking the SNR auction as an example, each user updates according to

$$
b_{i}(t)=f_{i}^{s}(\pi)\left(\sum_{j \neq i} b_{j}(t-1)+\beta\right) .
$$

To implement (33) directly, each user $i$ needs to know the sum of all other users' bids, which may not be possible in practice. However, we can show that (33) can be written in an equivalent form as

$$
b_{i}(t)=f_{i}^{s, d}(\pi, t-1) b_{i}(t-1),
$$

where (35) applies. It can be seen that $f_{i}^{s, d}(\pi)$ can be calculated locally by user $i$. When $\pi \in\left[\underline{\pi}_{i}^{s}, \hat{\pi}_{i}^{s}\right], f_{i}^{s, d}(\pi)$ depends on the difference between $\triangle \operatorname{SNR}_{i}\left(b_{i}(t-1), \boldsymbol{b}_{-i}(t-1)\right)$ and $\left(\frac{W}{2 \pi \ln 2}-1-\Gamma_{s_{i}, d_{i}}\right)$, and $f_{i}^{s, d}(\pi)$ does not equal 1 unless the two terms are the same. For the power auction, we can similarly show that the best response update can be written as

$$
b_{i}(t)=f_{i}^{p, d}(\pi, t-1) b_{i}(t-1),
$$

where $f_{i}^{p, d}(\pi, t-1)$ is similar to (35) (with more complicated notation) and is also locally computable.

In practice, users may not update their bids in each time slot. Therefore, we need to prove the convergence of the algorithm under asynchronous updates. The complete asynchronous best response update algorithm is given in Algorithm $1^{3}$, where

${ }^{3}[x]_{a}^{b}=\max \{\min \{x, b\}, a\} . f_{i}^{d}=f_{i}^{s, d}$ or $f_{i}^{p, d}$ depending on which auction the users participate in. 


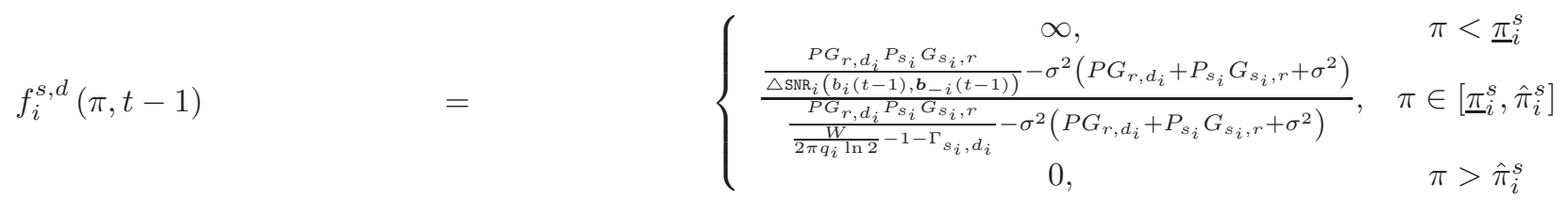

each user $i$ updates its bid only if the current time slot belongs to a set $\mathcal{T}_{i}$, which is an unbounded set of time slots. For example, $\mathcal{T}_{i}=\{1,3,7, \ldots\}$ means that user $i$ updates $b_{i}$ at time slot $1,3,7$, etc. We make a very mild assumption that the asynchronism of the updates is bounded, i.e., there exists a finite but sufficiently large positive constant $B$ (called the asynchronism measure [17, pp. 481]) such that

$$
\forall t_{1} \in \mathcal{T}_{i}, \exists t_{2} \in \mathcal{T}_{i}, t_{2}-t_{1} \leq B, \forall i \in \mathcal{I}
$$

From (37), each user updates its bid at least once during any time interval of length $B$ slots. The exact value of $B$ is not important and need not be known by the users.

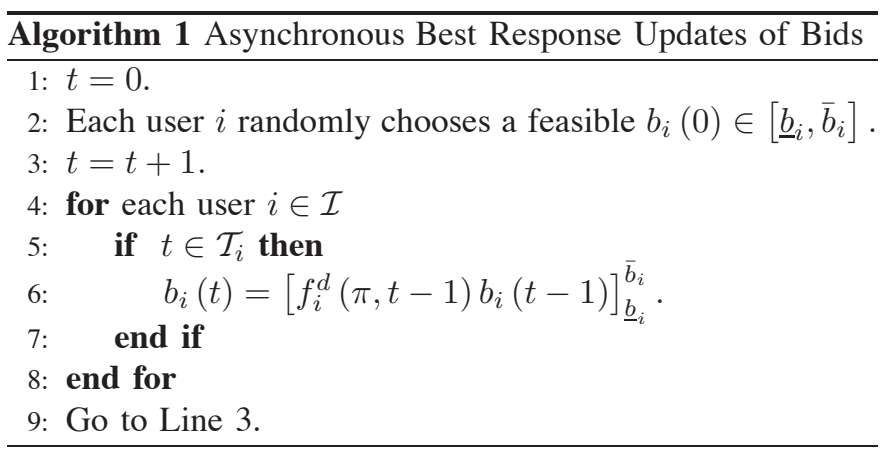

Theorem 7: If there exists a unique nonzero NE in the SNR or power auction, there always exists a lowerbound bid vector $\underline{\boldsymbol{b}}=\left\{\underline{b}_{i}\right\}_{i \in \mathcal{I}}$ and an upperbound bid vector $\overline{\boldsymbol{b}}=\left\{\bar{b}_{i}\right\}_{i \in \mathcal{I}}$, under which Algorithm 1 globally converges to the unique NE.

In practice, it is sufficient to choose $\underline{b}$ to be a sufficiently small and positive vector and $\bar{b}$ to be a sufficiently large and finite vector. To prove Theorem 7 , we can first verify that the best response update in (33) is in fact a standard interference function [19], and the rest of the proof is similar to that of Theorem 4 in [19].

\section{Extensions to Multiple Relay Networks}

The proposed auction-based resource allocation algorithms can be generalized to networks with multiple relays. Let us define the set of relays as $\mathcal{K}=\{1, \ldots, K\}$. Each relay $k \in \mathcal{K}$ announces a price $\pi_{k}$ and a reserve bid $\beta_{k}$, without knowing the prices and reserve bids of other relays. Each user $i \in \mathcal{I}$ submits a nonnegative bid vector $\boldsymbol{b}_{i}=\left\{b_{i k}\right\}_{k \in \mathcal{K}}$, one component for each relay. Based on the bids, relay $k$ allocates user $i$ with transmission power

$$
P_{r_{k}, d_{i}}=\frac{b_{i k}}{\sum_{j \in \mathcal{I}} b_{j k}+\beta_{k}} P_{k}
$$

where $P_{k}$ is the fixed total transmission power of relay $k$. This leads to an SNR increase of user $i$ of

$$
\triangle \mathrm{SNR}_{i k}=\Gamma_{s_{i}, r_{k}, d_{i}}=\frac{P_{r_{k}, d_{i}} P_{s_{i}} G_{r_{k}, d_{i}} G_{s_{i}, r_{k}}}{\sigma^{2}\left(P_{r_{k}, d_{i}} G_{r_{k}, d_{i}}+P_{s_{i}} G_{s_{i}, r_{k}}+\sigma^{2}\right)} .
$$

The total information rate that user $i$ achieves is

$$
R_{s_{i}, \boldsymbol{r}, d_{i}}=\frac{W \log _{2}\left(1+\Gamma_{s_{i}, d_{i}}+\sum_{k} \triangle \mathrm{SNR}_{i k}\right)}{\sum_{k} \mathbf{1}_{\left\{\triangle \mathrm{SNR}_{i k}>0\right\}}+1} .
$$

This includes a special case in which user $i$ does not use any relay (i.e., $\triangle \mathrm{SNR}_{i k}=0$ for all $k \in \mathcal{K}$ ). It is clear that $\triangle \mathrm{SNR}_{i k}>0$ if and only if $b_{i k}>0$. Notice that using more relays leads to higher total SNR increase $\sum_{k} \triangle \mathrm{SNR}_{i k}$, but also leads to a smaller coefficient $1 /\left(\sum_{k} \mathbf{1}_{\left\{\triangle \mathrm{SNR}_{i k}>0\right\}}+1\right)$. User $i$ needs to compare the total achievable rate under difference choices of relays, and choose one that yields the highest rate increase.

We first consider the SNR auction where user $i$ 's payment is $C_{i}=\sum_{k} \pi_{k} q_{i k} \triangle \mathrm{SNR}_{i k}$.

Theorem 8: In an SNR auction with multiple relays, a user $i$ either does not use any relay, or only uses one relay $r_{k(i)}$ with the smallest weighted price, i.e., $k(i)=\arg \min _{k \in \mathcal{K}} \pi_{k} q_{i k}$.

Theorem 8 can be proved by examining the first order conditions that need to be satisfied at the NE. In case there are multiple relays that announce the same smallest weighted price, user $i$ can randomly choose one of them. The choice of relay can be made by a user before knowing other users' bids. This implies that we can divide a multiple-relay network into $K+1$ clusters of nodes: each of the first $K$ clusters contains one relay node and the users who use this relay, and the last cluster contains users that do not use any relay. Then we can analyze each cluster independently as a single-relay network as in Section III-A.

Now consider the power auction where user $i$ 's payment is $C_{i}=\sum_{k} \pi_{k} P_{r_{k}, d_{i}}$. There are several key differences here compared with the SNR auction. First, a user may choose to use multiple relays simultaneously here. User $i$ 's best response can be written in the following linear form:

$$
\mathcal{B}_{i, k}\left(\boldsymbol{b}_{-i, k}, \boldsymbol{\pi}\right)=f_{i, k}^{p}(\boldsymbol{\pi})\left(\sum_{j \neq i} b_{j, k}+\beta_{k}\right), \forall k \in \mathcal{K} .
$$

There are two key differences here compared with the singlerelay power auction. First, to calculate $f_{i, k}^{p}(\boldsymbol{\pi})$, user $i$ needs to consider a total of $\sum_{l=0}^{K}\left(\begin{array}{c}K \\ l\end{array}\right)$ cases of choosing relays. For example, when there are two relays in the network, a user needs to consider four cases: not using any relay, using relay 1 only, using relay 2 only, and using both relays. For the given relay choice in case $n$, it calculates the linear coefficients $f_{i, k}^{p, n}(\boldsymbol{\pi})$ for all $k$ in closed-form (also involves threshold policy similar as in the SNR auction) and the 


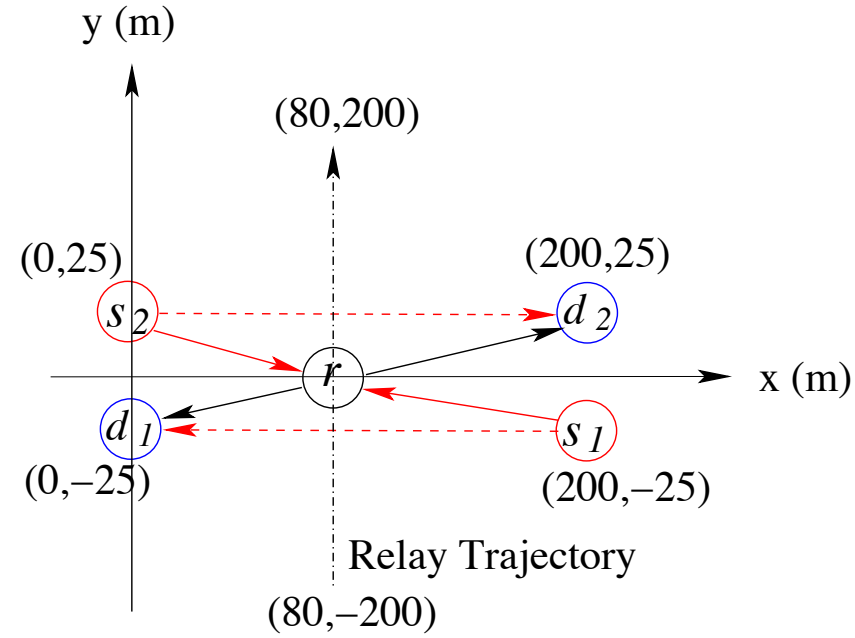

Fig. 4. A two-user cooperative network

corresponding rate increase $\triangle R_{i}^{n}$. Then it finds the case that yields the largest payoff, $n^{*}=\arg \max _{n} \triangle R_{i}^{n}$, and sets $f_{i, k}^{p}(\boldsymbol{\pi})=f_{i, k}^{p, n^{*}}(\boldsymbol{\pi}) \forall k$. Second, the linear coefficient $f_{i, k}^{p}(\boldsymbol{\pi})$ is dependent on the prices announced by all relays. For example, a large $\pi_{k}$ or a small $\pi_{k^{\prime}}\left(k^{\prime} \neq k\right)$ may lead to $f_{i, k}^{p}(\boldsymbol{\pi})=0$, i.e., user $i$ chooses not to use relay $r_{k}$.

Similar to the single relay power auction, we can also calculate a threshold price $\pi_{k, t h}^{p}$ for relay $r_{k}$. In this case, we assume that all relays except $r_{k}$ announce infinitely high prices, and then calculate $\pi_{k, t h}^{p}$ such that $\sum_{i \in \mathcal{I}} \frac{f_{i, k}^{p}\left(\pi_{k}\right)}{f_{i, k}^{p}\left(\pi_{k}\right)+1}<1$ when $\pi_{k}>\pi_{k, t h}^{p}$, and $\sum_{i \in \mathcal{I}} \frac{f_{i, k}^{p}\left(\pi_{k}\right)}{f_{i, k}^{p}\left(\pi_{k}\right)+1} \geq 1$ when $\pi_{k} \leq \pi_{k, t h}^{p}$.

Corollary 1: In a power auction with multiple relays, there exists an NE if $\pi_{k}>\pi_{k, t h}^{p}$ for each $k$.

On the other hand, necessary condition for the existence of an NE as well as conditions for uniqueness are not straightforward to specify, and are left for future research.

Finally, note that if there exists a unique NE in the SNR auction or power auction with multiple relays, the asynchronous best response updates of the users will globally converge to that NE.

\section{Simulation Results}

For illustration purposes, here we provide simulation results for a single relay network. We first simulate two auction mechanisms for a two-user network. As shown in Fig. 4, the locations of the two sources $\left(s_{1}\right.$ and $\left.s_{2}\right)$ and two destinations $\left(d_{1}\right.$ and $\left.d_{2}\right)$ are fixed at $(200 \mathrm{~m},-25 \mathrm{~m}),(0 \mathrm{~m}, 25 \mathrm{~m}),(0 \mathrm{~m},-25 \mathrm{~m})$, $(200 \mathrm{~m}, 25 \mathrm{~m})$. The $x$ coordinate of the relay node $r$ at $80 \mathrm{~m}$, and its $y$ coordinate varies within the range $[-200 \mathrm{~m}, 200 \mathrm{~m}]$. In the simulation, the relay moves along the dotted line. The propagation loss factor is set to 4 , and the channel gains are distance based (i.e., time-varying fading is not considered here). The transmit power between a source and its destination is $P_{i}=0.01 \mathrm{~W}$, the noise level is $\sigma^{2}=10^{-11} \mathrm{~W}$, and the total power of the relay node is $P=0.1 \mathrm{~W}$.

In Fig. 5, we show the individual rate increases of both users in both auctions. The weights in the SNR auction are chosen as $q_{i}=1$ for each user $i$. We first consider the power auction. The individual rate increases under the power auction are similar as

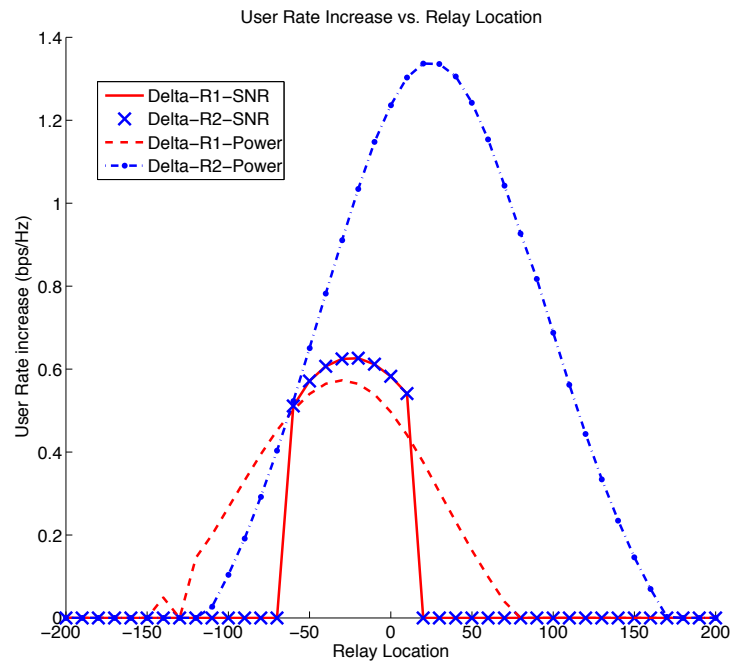

Fig. 5. Individual rate increase vs. relay location (y-axis) for an SNR auction and a power auction (users have equal weights in the SNR auction).

that achieved under a VCG auction (not shown here) and thus are efficient. Since the relay movement trajectory is relatively closer to source $s_{2}$ than to source $s_{1}$, user 2 achieves an overall better performance compared with user 1 . In particular, user 2 achieves a peak rate increase of $1.35 \mathrm{bps} / \mathrm{Hz}$ when the relay is at location $25 \mathrm{~m}$ (y-axis), compared with the peak rate increase of $0.56 \mathrm{bps} / \mathrm{Hz}$ achieved by user 1 when the relay is at location $-25 \mathrm{~m}$. Things are very different in the SNR auction, where the resource allocation is fair. In particular, since the distance between a source and its destination is the same for each of the users, both users achieve the same positive rate increases when they both use the relay. This is the case when the relay is between locations $-60 \mathrm{~m}$ and $10 \mathrm{~m}$. At other locations, users simply choose not to use the relay since they cannot both get equal rate increase while obtaining a positive payoff. This shows the tradeoff between efficiency and fairness.

In Fig. 6, we show the individual rate increases for two auctions, with the weights in the SNR auction set to $q_{1}=$ 0.5 and $q_{2}=1$. Here user 2 achieves a higher rate increase than user 1 in the SNR auction, due to its higher weight. By adjusting the weights of different users, the SNR auction can achieve different tradeoffs between fairness and efficiency.

Next, we consider the case where there are 20 users in the network, with their source nodes and destination nodes randomly and uniformly located within the square field that has the same range of $[-150 \mathrm{~m}, 150 \mathrm{~m}]$ on both the $x$-axis and the $y$-axis. A single relay is fixed at the location $(0 \mathrm{~m}, 0 \mathrm{~m})$. We change the total transmission power $P$ of the relay from 0.04 $\mathrm{W}$ to $1 \mathrm{~W}$. Figs. 7 and 8 show the corresponding simulation results. Each point in the figures represents the averaged result over 100 randomly generated network topologies. All users have the same weights in SNR auction. With an increasing amount of resource at the relay node, the total network rate increase improves in both auctions (Fig. 7), and the power auction achieves higher rate increase than the SNR auction. Fig. 8 shows the variance of the rate increase (among the users with positive rate increase), and it is clear that the SNR 


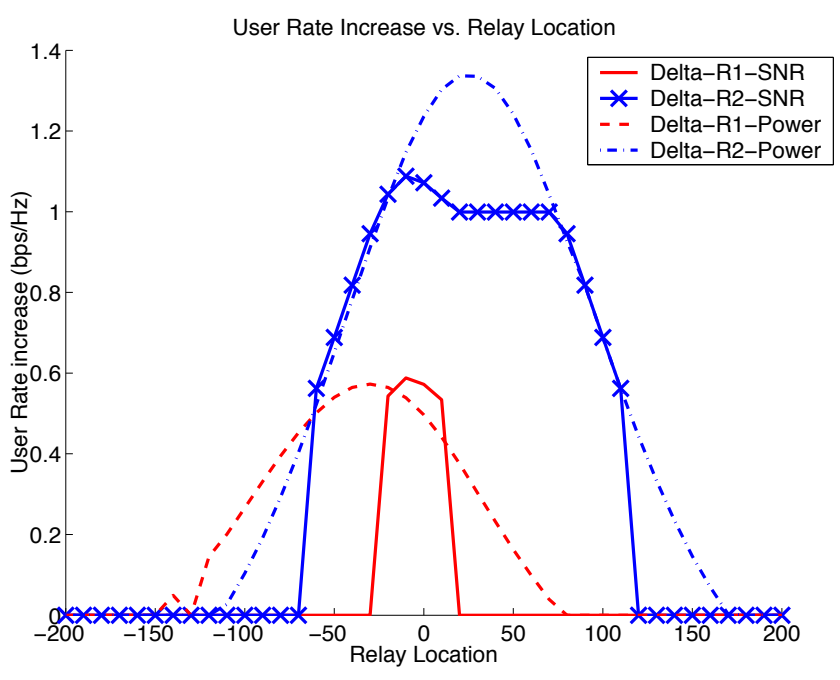

Fig. 6. Individual rate increase vs. relay location (y-axis) for an SNR auction and a power auction (users have different weights in the SNR auction).

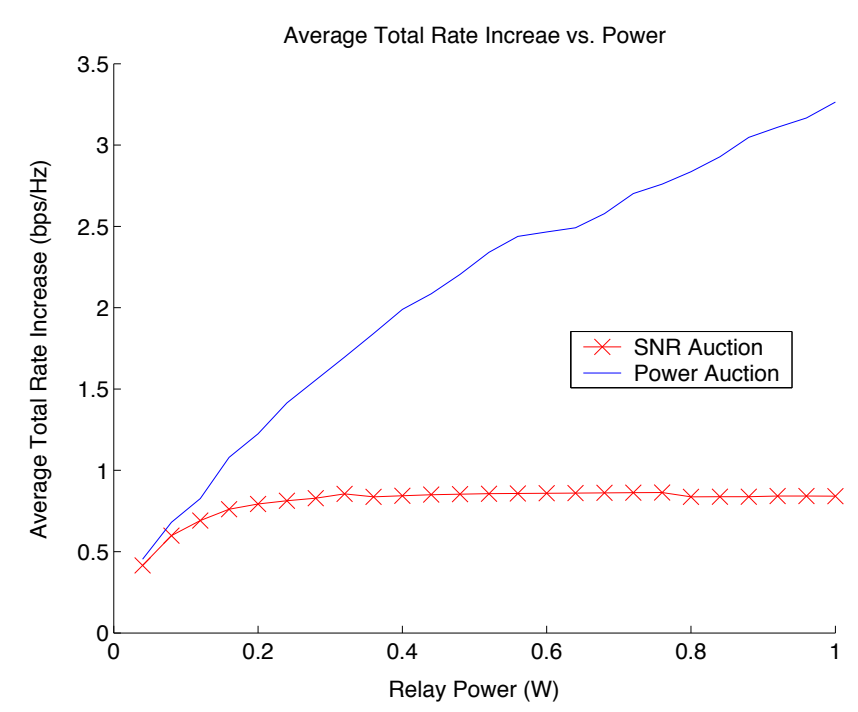

Fig. 7. Total network rate increase vs. relay power.

auction achieves a fairer resource allocation as indicated by the almost zero variance in all cases.

Finally, we show the convergence of Algorithm 1. We consider a three user network, where the three transmitters are located at $(100 \mathrm{~m},-25 \mathrm{~m}),(-100 \mathrm{~m}, 25 \mathrm{~m})$ and $(100 \mathrm{~m}, 5 \mathrm{~m})$, and the three receivers are located at $(-100 \mathrm{~m}, 25 \mathrm{~m}),(100 \mathrm{~m}, 25 \mathrm{~m})$ and $(-100 m, 5 m)$. The relay is located at $(0 \mathrm{~m},-2 \mathrm{~m})$. We simulate the SNR auction in this case. Three users randomly and independently choose to update their own bids in each time slot with probability $0.1,0.5$ and 1 , respectively. Convergence is clearly observed.

\section{CONCLUSIONS}

Cooperative transmission can greatly improve communication system performance by taking advantage of the broadcast nature of wireless channels and cooperation among users. In this paper, we have proposed two auction mechanisms, the SNR auction and the power auction, to distributively coordinate the relay power allocation among users. Under

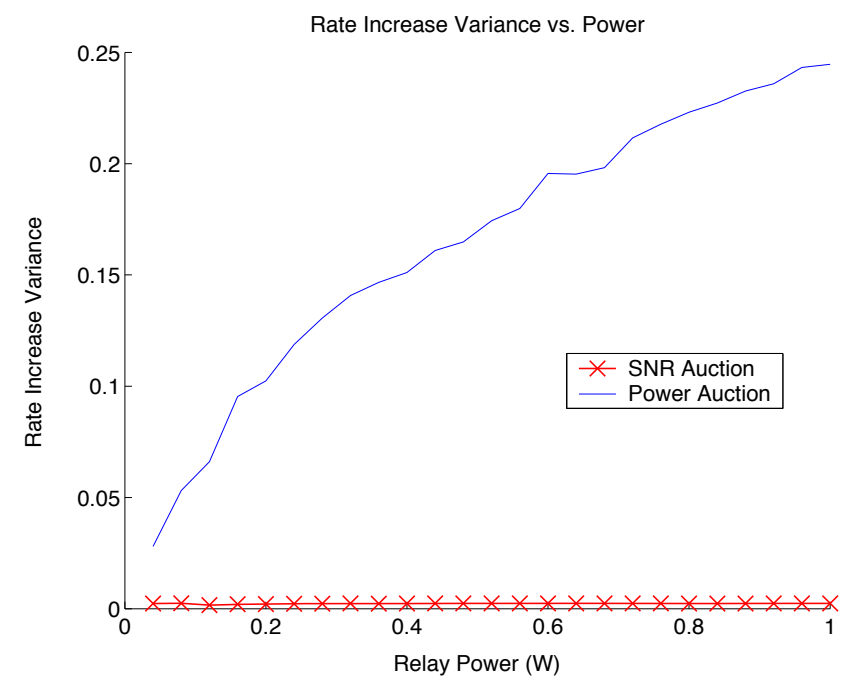

Fig. 8. Total positive rate increase variance vs. relay power.

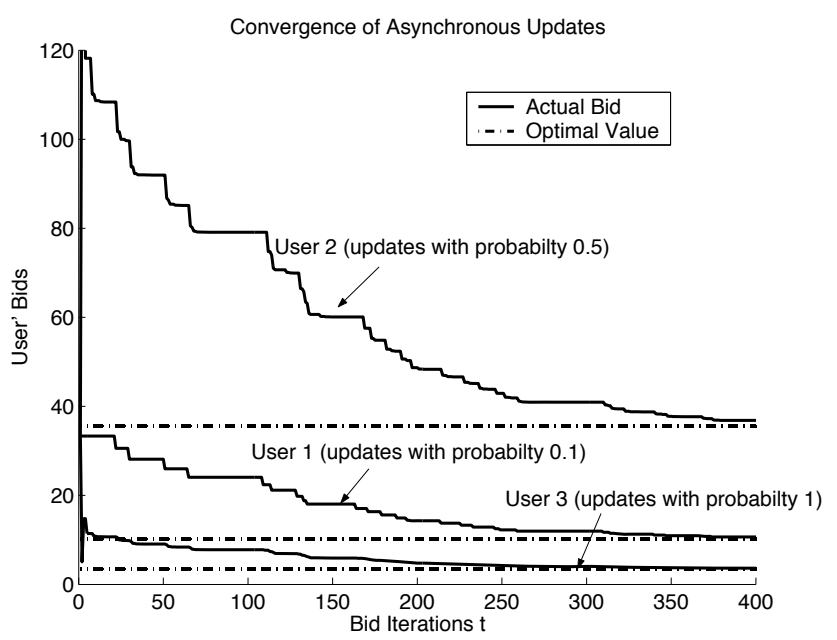

Fig. 9. Convergence of bids under asynchronous updates.

a fixed price announced by a single relay, we have shown that although each user has a non-smooth, non-concave utility function, its best response function can nevertheless explicitly be calculated locally based on a simple threshold policy. The existence and uniqueness of the Nash equilibrium in both auctions has been proved using nonnegative matrix theory. Furthermore, under properly chosen prices, the power auction has been shown to achieve the efficient allocation, and the SNR auction has been seen to be flexible in achieving various tradeoffs between fairness and efficiency depending on the priority weights. Furthermore, we have shown that users can achieve the unique NE in a completely distributed and asynchronous fashion. We have also shown that the main properties of the two auctions are applicable for networks with multiple relays at different locations.

\section{APPENDIX}

\section{A. Proof of Theorem 1}

In an SNR auction, user $i$ 's payoff function is

$U_{i}\left(b_{i}, \boldsymbol{b}_{-i}, \pi\right)=\triangle R_{i}\left(P_{r, d_{i}}\left(b_{i} ; \boldsymbol{b}_{-i}\right)\right)-\pi q_{i} \triangle \operatorname{SNR}_{i}\left(b_{i}, \boldsymbol{b}_{-i}\right)$, 


$$
\triangle \quad R_{i}\left(P_{r, d_{i}}\left(b_{i} ; \boldsymbol{b}_{-i}\right)\right) \quad \quad \quad \quad \quad \max \left\{\frac{W}{2} \log _{2}\left(1+\Gamma_{s_{i}, d_{i}}+\triangle \operatorname{SNR}_{i}\left(b_{i}, \boldsymbol{b}_{-i}\right)\right)-R_{s_{i}, d_{i}}, 0\right\}
$$

where (43) is true. The best response function is

$$
\mathcal{B}_{i}\left(\boldsymbol{b}_{-i}, \pi\right)=\arg \max _{b_{i} \geq 0} U_{i}\left(b_{i}, \boldsymbol{b}_{-i}, \pi\right) .
$$

The difficulty of finding $\mathcal{B}_{i}\left(\boldsymbol{b}_{-i}, \pi\right)$ is due mainly to the max operation in (43), which leads to non-smoothness and non-concavity of the payoff function. Next, we solve for $\mathcal{B}_{i}\left(\boldsymbol{b}_{-i}, \pi\right)$ in two steps.

First, by temporarily ignoring the max operation, we solve for the following modified best response function,

$$
\tilde{\mathcal{B}}_{i}\left(\boldsymbol{b}_{-i}, \pi\right)=\arg \max _{b_{i} \geq 0} \tilde{U}_{i}\left(b_{i}, \boldsymbol{b}_{-i}, \pi\right),
$$

where the modified payoff function is

$$
\begin{gathered}
\tilde{U}_{i}\left(b_{i}, \boldsymbol{b}_{-i}, \pi\right)=\frac{W}{2} \log _{2}\left(1+\Gamma_{s_{i}, d_{i}}+\triangle \operatorname{SNR}_{i}\left(b_{i}, \boldsymbol{b}_{-i}\right)\right) \\
-R_{s_{i}, d_{i}}-\pi q_{i} \triangle \operatorname{SNR}_{i}\left(b_{i}, \boldsymbol{b}_{-i}\right) .
\end{gathered}
$$

Differentiating $\tilde{U}_{i}\left(b_{i}, \boldsymbol{b}_{-i}, \pi\right)$ with respect to $b_{i}$, we have

$$
\begin{aligned}
& \frac{\partial \tilde{U}_{i}\left(b_{i}, \boldsymbol{b}_{-i}, \pi\right)}{\partial b_{i}}=\frac{\partial \triangle \operatorname{SNR}_{i}\left(b_{i}, \boldsymbol{b}_{-i}\right)}{\partial b_{i}} \\
& \quad \times\left(\frac{W}{2 \ln 2\left(1+\Gamma_{s_{i}, d_{i}}+\triangle \operatorname{SNR}_{i}\left(b_{i}, \boldsymbol{b}_{-i}\right)\right)}-\pi q_{i}\right) .
\end{aligned}
$$

It is clear that $\partial \triangle \operatorname{SNR}_{i}\left(b_{i}, \boldsymbol{b}_{-i}\right) / \partial b_{i}$ is strictly positive. Furthermore, the term in the brackets on the right hand side of (47) is strictly decreasing in $b_{i}$. If the price is appropriate such that there exists a $b_{i}$ so that the term in the brackets equals zero, we have found the best response. If the price is too high (or too low), the best response is 0 (or $\infty$ ). In summary, we can show that the unique best response $\tilde{\mathcal{B}}_{i}\left(\boldsymbol{b}_{-i}, \pi\right)$ in this case can be written as

$$
\tilde{\mathcal{B}}_{i}\left(\boldsymbol{b}_{-i}, \pi\right)=\tilde{f}_{i}^{s}(\pi)\left(\sum_{j \neq i} b_{j}+\beta\right),
$$

where the equation on the top of the following page is true. $\underline{\pi}_{i}^{s}$ is defined in (20) and $\bar{\pi}_{i}^{s}=1 /\left(2 q_{i} \ln 2\left(1+\Gamma_{s_{i}}^{D T}\right)\right)>\underline{\pi}_{i}^{s}$. As a result, we can calculate the maximum value of the modified payoff function, $\tilde{U}_{i}\left(\tilde{\mathcal{B}}_{i}\left(\boldsymbol{b}_{-i}, \pi\right), \boldsymbol{b}_{-i}, \pi\right)$, which turns out to depend only on the price $\pi$,

$$
\tilde{U}_{i}^{\max }(\pi)=\left\{\begin{array}{cc}
g_{i}^{s}\left(\underline{\pi}_{i}^{s}\right), & \pi<\underline{\pi}_{i}^{s} \\
g_{i}^{s}(\pi), & \pi \in\left[\underline{\pi}_{i}^{s}, \bar{\pi}_{i}^{s}\right] \\
g_{i}^{s}\left(\bar{\pi}_{i}^{s}\right), & \pi>\bar{\pi}_{i}^{s}
\end{array}\right.
$$

where the function $g_{i}^{s}(\cdot)$ is defined in (21).

Next, we consider the max operation in (43) and consider the original payoff function $U_{i}\left(b_{i} ; \boldsymbol{b}_{-i}, \pi\right)$ as in (42). We notice that user $i$ can always submit a zero bid and achieve a zero payoff. This implies that the difference between the best response function $\mathcal{B}_{i}\left(\boldsymbol{b}_{-i}, \pi\right)$ and the modified one $\tilde{\mathcal{B}}_{i}\left(\boldsymbol{b}_{-i}, \pi\right)$ occurs in the prices range where $\tilde{U}_{i}^{\max }(\pi)<0$. It can be verified that $g_{i}^{s}(\pi)$ is strictly decreasing over $\left(0, \bar{\pi}_{i}^{s}\right]$, with $g_{i}^{s}\left(\bar{\pi}_{i}^{s}\right)<0$ and $\lim _{\pi \rightarrow 0} g_{i}^{s}(\pi)>0$. Thus using bisection search, we can find a unique price $\hat{\pi}_{i} \in\left(0, \bar{\pi}_{i}^{s}\right)$ such that $g_{i}^{s}\left(\hat{\pi}_{i}^{s}\right)=0$. Then for any price $\pi>\hat{\pi}_{i}^{s}$, we have $\tilde{U}_{i}^{\max }(\pi)<0$, which implies that $\mathcal{B}_{i}\left(\boldsymbol{b}_{-i}, \pi\right)=0$. Together with the result in (49), we have proved the theorem.

\section{B. Proof of Theorem 2}

Based on (22), we know that an NE $\boldsymbol{b}^{*}$ is a nonnegative solution of the following set of linear equations:

$$
\left(\boldsymbol{I}-\boldsymbol{F}^{s}(\pi)\right) \boldsymbol{b}=\boldsymbol{f}^{s}(\pi) \beta,
$$

where $I$ is an $I \times I$ identity matrix, $\boldsymbol{F}^{s}(\pi)$ is an $I \times I$ matrix whose $(i, j)$ th component equals to $f_{i}^{s}(\pi)$ for $i \neq j$, and diagonal terms equal to zero, and $f^{s}(\pi)$ is an $I \times 1$ vector whose $i$ th component equals $f_{i}^{s}(\pi)$. It is clear that there exists a unique NE if and only if the spectral radius of the nonnegative matrix $\boldsymbol{F}^{s}(\pi)$ (defined as $\rho\left(\boldsymbol{F}^{s}(\pi)\right)$ ) is less than 1 , in which case the unique NE is $\boldsymbol{b}^{*}=\sum_{n=0}^{\infty}$ $\left(\boldsymbol{F}^{s}(\pi)\right)^{n} \boldsymbol{f}^{s}(\pi) \beta$. By using nonnegative matrix theory [18], we can show that $\rho\left(\boldsymbol{F}^{s}(\pi)\right)$ is a decreasing function of $\pi$, and we can find a threshold $\pi_{t h}^{s}$ such that $\rho\left(\boldsymbol{F}^{s}(\pi)\right)<1$ when $\pi>\pi_{t h}^{s}$, and $\rho\left(\boldsymbol{F}^{s}(\pi)\right) \geq 1$ otherwise. Details are omitted due to space limitations.

\section{Proof of Theorem 3}

Here we follow the notations used in the proof of Theorem 2. Since $f_{i}^{s}(\pi)$ is continuous at $\pi_{t h}^{s}$ for all users, we can show that $\rho\left(\boldsymbol{F}^{s}(\pi)\right)$ is also continuous at $\pi_{t h}^{s}$. Moreover, since $f_{i}^{s}(\pi)$ is positive and finite for at least one user, we can show that $\rho\left(\boldsymbol{F}^{s}(\pi)\right)$ can be made arbitrarily close but smaller than 1 with properly chosen price. This means that the summation $\sum_{i} b_{i}^{*}$ (where $\boldsymbol{b}^{*}=\sum_{n=0}^{\infty}\left(\boldsymbol{F}^{s}(\pi)\right)^{n} \boldsymbol{f}^{s}(\pi) \beta$ is the unique $\mathrm{NE}$ ) can be made arbitrarily large, and the resource waste factor $\beta P /\left(\sum_{i} b_{i}^{*}+\beta\right)$ can be made arbitrarily small.

\section{Proof of Proposition 1}

Let us define the modified payoff function as

$$
\begin{array}{r}
\tilde{U}_{i}\left(P_{r, d_{i}}, \pi\right)=\frac{W}{2} \log _{2}\left(1+\Gamma_{s_{i}, d_{i}}+\triangle \operatorname{SNR}_{i}\left(P_{r, d_{i}}\right)\right) \\
-R_{s_{i}, d_{i}}-\pi P_{r, d_{i}} .
\end{array}
$$

We can show that the unique value of $P_{r, d_{i}}$ that maximizes $\tilde{U}_{i}\left(P_{r, d_{i}}\right)$ is

$P_{r, d_{i}}^{*}(\pi)=\arg \max _{P_{r, d_{i}} \geq 0} \tilde{U}_{i}\left(P_{r, d_{i}}, \pi\right)=\frac{z_{i}(\pi)}{G_{r, d_{i}}}, \forall \pi \in\left[\underline{\pi}_{i}^{p}, \bar{\pi}_{i}^{p}\right]$,

with $P_{r, d_{i}}^{*}\left(\underline{\pi}_{i}^{p}\right)=P$ and $P_{r, d_{i}}^{*}\left(\bar{\pi}_{i}^{p}\right)=0$. Furthermore, $g_{i}^{p}(\pi)=\tilde{U}_{i}\left(P_{r, d_{i}}^{*}(\pi), \pi\right)$.

Assume that $g_{i}^{p}(\pi)$ is not strictly decreasing with $\pi \in$ $\left[\underline{\pi}_{i}^{p}, \bar{\pi}_{i}^{p}\right]$. Then there exists a $\pi^{\prime}<\pi^{\prime \prime}$, such that $g_{i}^{p}\left(\pi^{\prime}\right) \leq$ 


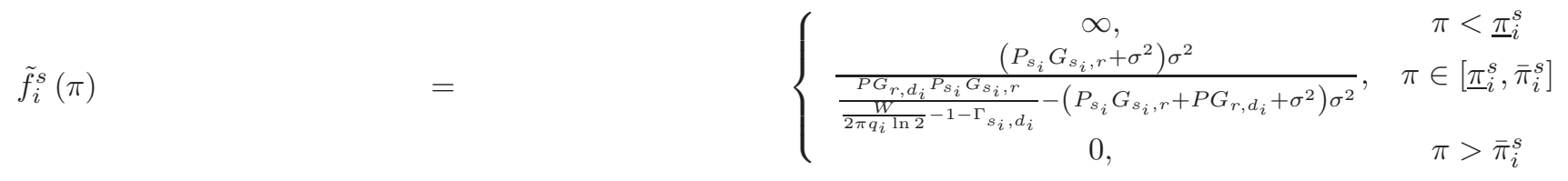

$g_{i}^{p}\left(\pi^{\prime \prime}\right)$, i.e, $\tilde{U}_{i}\left(P_{r, d_{i}}^{*}\left(\pi^{\prime}\right), \pi^{\prime}\right) \leq \tilde{U}_{i}\left(P_{r, d_{i}}^{*}\left(\pi^{\prime \prime}\right), \pi^{\prime \prime}\right)$. However, from (51) and the optimality of $P_{r, d_{i}}^{*}(\pi)$ we know that $\tilde{U}_{i}\left(P_{r, d_{i}}^{*}\left(\pi^{\prime}\right), \pi^{\prime}\right) \geq \tilde{U}_{i}\left(P_{r, d_{i}}^{*}\left(\pi^{\prime \prime}\right), \pi^{\prime}\right)>$ $\tilde{U}_{i}\left(P_{r, d_{i}}^{*}\left(\pi^{\prime \prime}\right), \pi^{\prime \prime}\right)$, which leads to a contradiction. This completes the proof.

\section{REFERENCES}

[1] A. Sendonaris, E. Erkip, and B. Aazhang, "User cooperation diversity - Part I: System description," IEEE Transactions on Communications, vol. 51, no. 11, pp. 1927-1938, November 2003.

[2] J. Laneman, D. Tse, and G. Wornell, "Cooperative diversity in wireless networks: Efficient protocols and outage behavior," IEEE Transactions on Information Theory, vol. 50, no. 12, pp. 3062-3080, December 2004.

[3] T. Cover and A. El-Gamal, "Capacity theorems for the relay channel," IEEE Transactions Information Theory, vol. 25, no. 5, pp. 572-584, September 1979.

[4] M. A. Khojasterpour, A. Sabharwal, and B. Aazhang, "On the capacity of 'cheap' relay networks," Proceedings of the Conference Information Sciences and Systems, Baltimore, MD, March 2003.

[5] T. Hunter and A. Nosratinia, "Diversity through coded cooperation," IEEE Transactions on Wireless Communications, vol. 5, no. 2, pp. 283289, Feb. 2006.

[6] A. Nosratinia and T. E. Hunter, "Grouping and partner selection in cooperative wireless networks," IEEE Journal on Selected Areas in Communications, vol 25, no. 2, pp. 369-378, February 2007.

[7] T. Ng and W. Yu, "Joint optimization of relay strategies and resource allocations in cooperative cellular networks," IEEE Journal on Selected Areas in Communications, vol 25, no. 2, pp. 328-339, February 2007.

[8] A. Bletsas, A. Lippman, and D. P. Reed, "A simple distributed method for relay selection in cooperative diversity wireless networks, based on reciprocity and channel measurements", Proceedings of the IEEE Vehicular Technology Conference Spring, Stockholm, Sweden, May 2005.

[9] S. Savazzi and U. Spagnolini, "Energy aware power allocation strategies for multihop-cooperative transmission schemes," IEEE Journal on Selected Areas in Communications, vol 25, no. 2, pp. 318-327, February 2007.

[10] T. Himsoon, W. Siriwongpairat, Z. Han, and K. J. R. Liu, "Lifetime maximization framework by cooperative nodes and relay deployment in wireless networks", IEEE Journal on Selected Areas in Communications, vol. 25, no. 2, p.p. 306-317, February, 2007.

[11] R. Annavajjala, P. C. Cosman, and L. B. Milstein, "Statistical channel knowledge-based optimum power allocation for relaying protocols in the high SNR regime," IEEE Journal on Selected Areas in Communications, vol 25, no. 2, pp. 292-305, February 2007.

[12] B. Wang, Z. Han, and K. J. R. Liu, "Distributed Relay Selection and Power Control for Multiuser Cooperative Communication Networks Using Buyer / Seller Game", Proceedings of IEEE INFOCOM, Anchorage, AK, May 2007.

[13] V. Krishna, Auction Theory, Academic Press, London, UK, 2002.

[14] J. Sun, E. Modiano, and L. Zheng, "Wireless channel allocation using an auction algorithm," IEEE Journal on Selected Areas in Communications, vol. 24, no. 5, 2006, pp. 1085-1096, May 2006.

[15] M. Dramitinos, G. Stamoulis, and C. Courcoubetis, "Auction-based resource reservation in 2.5/3G networks," Mobile Networks and Applications, vol. 9, no. 6, pp. 557-566, December 2004.

[16] J. Huang, R. Berry, and M. L. Honig, "Auction-based spectrum sharing," Mobile Networks and Applications, vol. 11, no. 3, pp. 405-418, June 2006.

[17] D. P. Bertsekas and J. N. Tsitsiklis, Parallel and Distributed Computation: Numerical Methods, Prentice Hall, Englewood Cliffs, NJ, 1989.

[18] R. A. Horn and C. R. Johnson, Matrix Analysis, Cambridge University Press, Cambridge, UK, 1985.
[19] R. D. Yates, "A framework for uplink power control in cellular radio systems," IEEE Journal on Selected Areas in Communications, vol. 13, no. 7, pp. 1341-1347, September 1995.

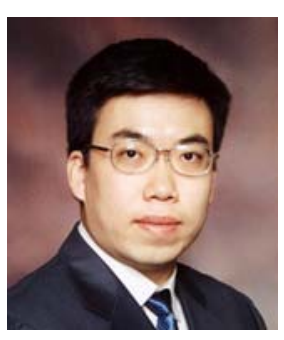

Jianwei Huang (S'01-M'06) is an Assistant Professor in Information Engineering Department at the Chinese University of Hong Kong. He received the M.S. and Ph.D. degrees in Electrical and Computer Engineering from Northwestern University (Evanston, IL, USA) in 2003 and 2005, respectively. From 2005 to 2007, He worked as a Postdoctoral Research Associate in the Department of Electrical Engineering at Princeton University (Princeton, NJ, USA). In 2004 and 2005, he worked in the Mathematics of Communication Networks Group at Motorola (Arlington Heights, IL, USA) both as a full time summer intern and a part time researcher. In 1999, he worked as a summer intern in the Department of Change Management at GKN Westland Aerospace (Cowes, Isle of Wight, UK). His main research interests lie in the area of modeling and performance analysis of communication networks, with specific areas including cognitive radio networks, wideband OFDM and CDMA systems, wireless medium access control, multimedia communications, network economics, and applications of optimization theory and game theory.

Dr. Huang is an Associate Editor of (Elsevier) Journal of Computer \& Electrical Engineering, the Lead Guest Editor of the IEEE Journal of Selected Areas in Communications special issue on "Game Theory in Communication Systems", the Lead Guest Editor of the Journal of Advances in Multimedia special issue on "Collaboration and Optimization in Multimedia Communications", and a Guest Editor of the Journal of Advances in Multimedia special issue on "Cross-layer Optimized Wireless Multimedia Communications". He will be the Technical Program Committee Co-Chair of the International Conference on Game Theory for Networks (GameNets'09), and is a TPC member for many conferences such as IEEE INFOCOM, GlobeCom, WCNC, ICCCN, CCNC, and CrownCom. Dr. Huang is the recipient of a 2001 Walter P. Murphy Fellowship at Northwestern University and a 1999 Chinese National Excellent Student Award. 


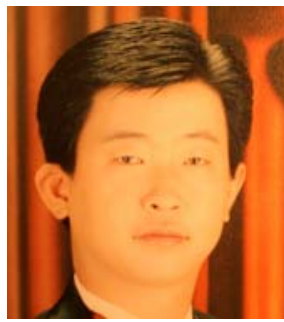

Zhu Han (S'01 -M'04) received the B.S. degree in electronic engineering from Tsinghua University, in 1997, and the M.S. and Ph.D. degrees in electrical engineering from the University of Maryland, College Park, in 1999 and 2003, respectively.

From 2000 to 2002, he is an R\&D Engineer of JDSU, Germantown, Maryland. From 2002 to 2003, he was a Graduate Research Assistant at the University of Maryland. From 2003 to 2006, he was a Research Associate at the University of Maryland. From 2006 to 2008, he was an assistant professor in Boise State University, Idaho. Currently, he is an Assistant Professor in Electrical and Computer Engineering Department at University of Houston, Texas. In June-August 2006, he was a visiting scholar in Princeton University. In May-August 2007, he was a visiting professor in Stanford University. In May-August 2008, he was a visiting professor in University of Oslo, Norway and Supelec, Paris, France. His research interests include wireless resource allocation and management, wireless communications and networking, game theory, wireless multimedia, and security.

Dr. Han is the MAC Symposium vice chair of IEEE Wireless Communications and Networking Conference, 2008. Dr. Han is the Guest Editor for Special Issue on Fairness of Radio Resource Management Techniques in Wireless Networks, EURASIP Journal on Wireless Communications and Networking, and Special Issue on Game Theory, EURASIP Journal on Advances in Signal Processing. Dr. Han is a member of the Technical Programming Committee for the IEEE International Conference on Communications, the IEEE Vehicular Technology Conference, the IEEE Consumer Communications and Networking Conference, the IEEE Wireless Communications and Networking Conference, and the IEEE Globe Communication Conference.

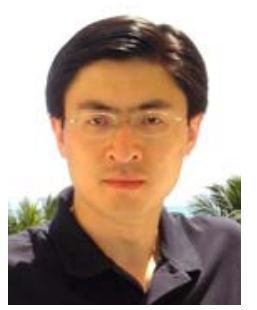

Mung Chiang (S'00-M'03) is an Associate Professor of Electrical Engineering and an affiliated faculty of Applied and Computational Mathematics and of Computer Science at Princeton University. $\mathrm{He}$ received the B.S. (Honors) in Electrical Engineering and Mathematics, M.S. and Ph.D. degrees in Electrical Engineering from Stanford University in 1999,2000 , and 2003, respectively.

He conducts research in the areas of optimization, distributed algorithm, and stochastic model of communication network, with applications to content distribution, broadband access, wireless networks, and the Internet. $\mathrm{He}$ received CAREER Award from the National Science Foundation, Young Investigator Award from the Office of Naval Research, Howard B. Wentz Junior Faculty Award and Engineering Teaching Commendation from Princeton University, School of Engineering Terman Award from Stanford University, and was a Hertz Foundation Fellow. For his work on broadband access networks and Internet traffic engineering, he was selected for the TR35 Young Technologist Award in 2007, a list of top 35 innovators in the world under the age of 35. His work on Geometric Programming was selected by Mathematical Programming Society as one of the top 3 papers by young authors in the area of continuous optimization during 2004-2007. His work on Layering As Optimization Decomposition became a Fast Breaking Paper in Computer Science by ISI citation. He also co-authored papers that were IEEE INFOCOM best paper finalist and IEEE GLOBECOM best student paper. He has served as an associate editor for IEEE Transactions on Wireless Communications, an associate editor for Springer Journal on Optimization and Engineering, a lead guest editor for IEEE Journal of Selected Areas in Communications, a guest editor for IEEE/ACM Transactions on Networking and IEEE Transactions on Information Theory, a Program Co-Chair of the 38th Conference on Information Sciences and Systems, and a co-editor of the new Springer book series on "Optimization and Control of Communication Systems".

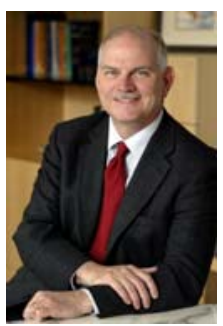

H. Vincent Poor (S'72-M'77-SM'82-F'87) received the Ph.D. degree in EECS from Princeton University in 1977. From 1977 until 1990, he was on the faculty of the University of Illinois at Urbana-Champaign. Since 1990 he has been on the faculty at Princeton, where he is the Michael Henry Strater University Professor of Electrical Engineering and Dean of the School of Engineering and Applied Science. Dr. Poor's research interests are in the areas of stochastic analysis, statistical signal processing and their applications in wireless networks and related fields. Among his publications in these areas is the recent book MIMO Wireless Communications (Cambridge University Press, 2007), co-authored with Ezio Biglieri, et al.

Dr. Poor is a member of the National Academy of Engineering, a Fellow of the American Academy of Arts and Sciences, and a former Guggenheim Fellow. He is also a Fellow of the Institute of Mathematical Statistics, the Optical Society of America, and other organizations. In 1990, he served as President of the IEEE Information Theory Society, and in 2004-07 he served as the Editor-in-Chief of the IEEE Transactions on Information Theory. Recent recognition of his work includes the 2005 IEEE Education Medal, the 2007 IEEE Marconi Prize Paper Award, and the 2007 Technical Achievement Award of the IEEE Signal Processing Society. 